\title{
The Role of Novel Coronavirus Pneumonia Outbreak Maps on the Internet in the Prediction and Early Warning of Infectious Diseases
}

\section{yufang Cao}

Affiliated Haikou Hospital of Xiangya Medical College, Central South University, Haikou, Hainan. https://orcid.org/0000-0003-4467-7032

Rui Qiu

Beijing Credithc Investment Management Co. LTD

Chao Ji

Affiliated Haikou Hospital of Xiangya Medical College, Central South University, Haikou, Hainan,

\section{Zhidian Wu}

The Second Affiliated Hospital of Hainan Medical University Haikou, Hainan

\section{Yijun Yang ( $\sim$ hksyy168@126.com )}

Central South University https://orcid.org/0000-0001-5978-328X

\section{Yingai Zhang}

Affiliated Haikou Hospital of Xiangya Medical College, Central South University, Haikou, Hainan,

\section{Research}

Keywords: Geoinformatics, COVID-19, map, GIS

Posted Date: November 19th, 2020

DOl: https://doi.org/10.21203/rs.3.rs-108782/v1

License: (9) This work is licensed under a Creative Commons Attribution 4.0 International License. Read Full License 


\section{Abstract}

\section{Background}

A novel coronavirus (2019-nCOV) has attracted worldwide attention since December 2019 when it appeared in Wuhan, China. The coronavirus disease (COVID-19) can cause respiratory distress syndrome or multiple organ dysfunction syndrome and be life-threatening. Covid-19 incidence can be reduced by using an outbreak map based on Geoinformatics.

\section{Methods}

This article introduces geographic mapping on the Internet and uses epidemic maps such as rank circles, choropleth rendering, overlay analysis and animation and other technologies to guide people's behavior, reduce entry into areas with severe epidemics, and prevent new crowd infections.

\section{Results}

By using various epidemic maps, households can reduce the access of people to the epidemic area (especially travellers to potentially at-risk areas), reduce the possibility of COVID-19 infection, and facilitate the timely diagnosis and treatment of fevered patients or suspected COVID-19.

In addition, public health managers can intuitively understand the dynamics of the epidemic and spatial analysis of trends in outbreak dynamics and COVID-19 patterns helped to assist the public health sector and to evaluate/revise current control measures.

\section{Conclusion}

The purpose of these online epidemic data collect and visualize is to notify travellers and assist the public or to analyzing the spatial and current trends and patterns of COVID-19 for public health authorities in assessing/revising current control measures.

\section{Introduction}

COVID-19 is caused by the coronavirus pathogen, and it is currently the main infectious disease worldwide. A group of patients with unexplained pneumonia in Wuhan, Hubei Province, China, was confirmed to be infected with a novel coronavirus in December 2019, namely, 2019-nCOV, that was not found in humans or animals previously. The World Health Organization (WHO) recently named the resulting disease, which has become a global problem, COVID-19. This is an atypical form of pneumonia that is highly infectious and potentially lethal and which begins with deceptive common flu-like symptoms. People usually have symptoms like fever, cough, or both at the beginning of infection. If the disease cannot be controlled in time, it will become further exacerbated, cause severe pulmonary infection and even respiratory failure that may be life-threatening[1,2]. Similar to the case for other coronavirus infections, the COVID-19 pneumonia has been confirmed of human-to-human transmission in case reports[3]. Since then, the disease has quickly spread along international routes[4], and the 
exponential growth of COVID-19 cases prompted the WHO to issue a warning to the world in January 2020[5]. From the end of 2019 to the first half of 2020, when the epidemic was at its worst, COVID-19 negatively affected all aspects of people's daily life, such as society, economy, transportation, work, tourism, school and family[6,7]. In December 2019, the WHO announced that it was not calling for restrictions on travel anywhere but would advise individuals to delay travel to dangerous areas until a later date.

Perhaps there are few things more inherently "geographical" than the epidemic spread study and control of a variety of appropriate scales. Geoinformatics is the science and technology of gathering, storing, visualizing, analyzing, interpreting, modelling, distributing and using spatially referenced (geographically referenced) information[8]. Geoinformatics plays an significant role in the research and efficient control of epidemics, from geographic mapping to epidemiological modelling and location-based data visualized alerting. This also applies equally to the global outbreak of COVID-19 in 2019. During the COVID-19 outbreak, the general public, travelers and public health decision-makers can use a carefully planned and designed online epidemic map to understand the epidemic situation quickly, avoid entering severe outbreak areas, and reduce the chance of infection. When designing and implementing epidemic control strategies or issuing and updating travel advisories and advice to the general public, such type of support is vital for making effective decisions.

\section{Materials And Methods}

First, users and health decision-makers can conveniently access the latest epidemic data as quickly as possible by visiting the COVID-19 Dashboard by the Center for Systems Science and Engineering (CSSE) at Johns Hopkins University (JHU) at https://coronavirus.jhu.edu/map.html. The data are based on the World Health Organization (WHO), Centers for Disease Control (CDC) of the United States, and CDC of China. Secondly, users can visit the WHO epidemic website online at https://covid19.who.int/ to understand the epidemic situation in various countries and regions around the world and analyze the trends of the epidemic. The data are reported by national health institutions and Centers for Disease Control and Prevention in each country. The JHU's COVID-19 Dashboard and the WHO's epidemic map include data on the number of confirmed, dead and cured cases in countries around the world as well as data charts and bars. The global epidemic map is easy to use. Furthermore, people can also download the Baidu Map app on their mobile phones in China and search for outbreaks using keywords of the surrounding areas. To prevent or reduce the number of health personnel entering areas with severe outbreaks, patients with a fever can also conveniently find a fever clinic for timely diagnosis and treatment as soon as possible.

\section{Results}

We can access the epidemic map to understand the global epidemic and control status to guide our work and life. Here are the results of a dynamic online observation of COVID-19. 


\section{Web-based COVID-19 datasets and maps: availability and features}

In this article, we review several cartographic charts of COVID-19 on the Internet. The Internet is the only source of reliable and real-time information on COVID-19 offered by the WHO and other official health bodies. It constitutes of the latest COVID-19 case counts and lists of affected areas etc. which reviewed in this article (Table).

Table Examples of frequently updated COVID-19 datasets on the Internet (WHO, CDC, and China Department of Health)*

URL Description

https://covid19.who.int/

Number of confirmed cases of COVID-19 globally

https://covid19.who.int/

Global death toll from COVID-19

https://coronavirus.jhu.edu/map.html

Number of confirmed and death cases of COVID19 globally

https://covid19.who.int/region/amro/country/us Number of confirmed COVID-19 patients in the United States

https://covid19.who.int/region/amro/country/us Deaths from COVID-19 in the United States

https://covid19.who.int/region/wpro/country/cn Number of confirmed COVID-19 patients in China https://covid19.who.int/region/wpro/country/cn Deaths from COVID-19 in China

*The table contains websites with WHO and Johns Hopkins University epidemic map data. You can check the website for information on the situation of the global epidemic.

\section{Map of COVID-19 outbreaks}

\section{Examples of geographic mapping of COVID-19 by the WHO}

Regarding an example of geographic mapping by the WHO for COVID-19, when COVID-19 emerged in December 2019, the WHO began to publish a daily map of the world's outbreaks and total number of reported possible cases on its world map (COVID-19 website). Examples of these epidemic maps are available from https://covid19.who.int/. On these maps, areas and countries that have not been affected by COVID-19 are marked in white, and countries and areas that have been affected by COVID-19 are marked in blue (Fig.1). On these epidemic maps, the cumulative number of reported cases is reflected by the size of the blue circles in the affected country/region (the larger the circles indicate more COVID-19 cases), and the number of infected cases can be displayed at the location selected by the cursor. The number of deaths is represented in orange, and an epidemic map of the number of deaths is also drawn (Fig.2) at https://covid19. who.int/. A larger circle indicates more cases, and a convenient toolbar provides the functionalities of the standard Arc View GIS desktop interface (for example, pan out, mark, zoom in, 
zoom out, measure, display the specific number of infections or deaths, etc.) as well as histograms and trend graphs of various countries for COVID-19 (Fig.3 and Fig.4).

\section{Examples of geographic mapping of COVID-19 by Baidu, Inc.}

In addition, in view of the novel pneumonia, Baidu, Inc. (Baidu), has an emergency online China outbreak map and maps of fever clinics and community outbreaks based on the official data of the Chinese Health Committee; users have the ability to click in the app "layer - medical map - fever clinics or epidemic village" to enter and view all nearby fever outpatient services of medical institutions (Fig.5). This information helps individuals avoid going to the wrong hospital and delaying treatment. Through the Baidu heat map at http://i7q.cn/6pXt24, the real-time population flow density of the region can be seen, and individuals can thus try to avoid going to business districts, transportation hubs and other crowded places. The upgraded Baidu "epidemic area" thematic map (Fig.6), after waiting for the user to enter, can be based on a user location intelligent popup "surrounding epidemic card" showing the number of epidemic sites in the current city (http://i7q.cn/6xtFZv), the number of epidemic sites within $1 \mathrm{~km}$, the number of epidemic sites within $3 \mathrm{~km}$, the name of the nearest epidemic site and the crowd gathering places of the epidemic site as well as other multidimensional real-time information briefings to facilitate users in quickly understanding the situation of the surrounding epidemic and performing epidemic prevention work in a timely manner. As of February 12, 2020, Baidu has completed an online "epidemic area" map of more than 200 cities. On the other hand, during the critical period of epidemic prevention and control, knowing the detailed data sources of the communities with confirmed cases and the places where people gather is also necessary.

\section{Examples of geographic mapping of COVID-19 by Johns Hopkins University}

Finally, one of the most famous outbreak maps is the COVID-19 dashboard developed by the Center for Systems Science and Engineering (CSSE) at Johns Hopkins University (JHU) https://coronavirus.jhu.edu/map.html. JHU is home to some of the world's top schools of medicine, public health, bioengineering and space sciences. With the novel coronavirus becoming viral around the world, transparent and real epidemic data are drawing much attention (Fig.7). JHU's real-time epidemic map stands out among many real-time visualization data release platforms and has become the main channel for countries to obtain epidemic data. Seeing the rising numbers on the map of the epidemic may play a role in keeping people at home. This is the most effective way to stop the spread of infectious diseases.

\section{Discussion}

\section{The role of geoinformatics in epidemics}

Since the first maps were used by Dr. John Snow in 1854 to trace the origin of a cholera outbreak in London's Soho district, carefully planned and designed maps have evidently been very powerful decision support and spatiotemporal analysis tools[9]. Geoinformatics is the science and technology of collecting, 
storing, analyzing, interpreting, visualizing, modeling, distributing and applying spatial reference information (GIS)[10]. Geoinformatics plays an important role in epidemiological research and response during epidemics, from geographic mapping to epidemiological modeling and location-based alert services[11,12]. These aspects also apply to the global outbreak of COVID-19 in 2019 (Table). During the global outbreak of COVID-19, WHO, JHU and Baidu used science and technology such as geoinformatics and computers to comprehensively map the epidemic. Outbreak maps are useful to the general public, travelers, and public health policy-makers and at-risk local residents as visual surveillance to monitor the trend of COVID-19 and patterns over time so as to make the right judgments and avoid entering serious epidemic areas, providing a dynamic basis for health policy makers to adjust schemes for controlling the outbreak. This support is vital for publishing and updating travel restriction or designing and following epidemic control strategies when making informed decisions .

\section{The function, characteristics and role of the epidemic map during the COVID-19 outbreak}

In the era of the Internet, COVID-19 is the world's leading infectious disease, and it takes full advantage of international air travel to spread rapidly. Geoinformatics plays a significant role in the research and control of global outbreaks, such as COVID-19, from geographic mapping to location-based alerting services. Thus the carefully designed maps based on big data collection and visualization should be the most powerful decision support and spatiotemporal analysis tools.

The epidemic map intuitively expresses the changes and current situation of the epidemic situation. It is an interweaving and collision of database science and surveying and mapping science. By studying the spatial distribution of patients, we can assess the epidemic situation, transmission mode and epidemic process and determine the prevention and control measures of infectious diseases through policies. The COVID-19 outbreak map website can be visited for global data on active cases, convalescence, morbidity, mortality (\%), and so on. The Johns Hopkins Coronavirus Resource Center allows experts to help advance understanding of the virus, inform the public, and advise policymakers in order to implement effective measure, improve care, and save lives. By visiting Baidu epidemic map in China, we can not only understand the epidemic situation around us but also guide patients to the nearest fever clinic in a timely manner so as not to delay treatment.

Compared with other publishing media, web-based online maps also allow for interactivity to be incorporated into the maps (desktop-like GIS functionality, e.g., drilling down and zooming), and for more wider and rapid dissemination of information, besides the well-known feature of frequent, real-time updates based on the latest datasets. In this paper, we have reviewed several geographic mapping efforts for COVID-19 on the Internet. A variety of techniques, such as graduated pie charts, graduated circles, choropleth rendering, thematic mapping, buffering, overlay analysis and animation, were employed in the maps described in this article, to visually monitor and pay attention to the patterns, trends and changes with time hidden in large datasets (such as the COVID-19 dataset) for public health decision-makers, travelers and local populations at risk. 
In addition, the COVID-19 Dashboard developed by Johns Hopkins University has the characteristics of truthful, transparent and timely updated data, thus becoming the main channel for countries to obtain epidemic data. This article provides some detailed mapping information down to each subdivision of the major cities by Baidu. Therefore, as described in this paper, web-based maps provide the following informatization features absent in traditional paper-based maps: (1) interactivity (for example, map querying, drilling down and scaling, measuring distances, and so on); (2) a map that can be updated quickly and frequently according to the latest dataset; (3) a map that is real, transparent, intuitive and convenient; and (4) wide dissemination to a large audience.

This study describes the mapping of COVID-19 outbreaks based on geographic information science combined with multidisciplinary related technologies widely used in the global outbreak of COVID-19. The widespread use of epidemic maps is critical to raising global awareness on an overall level and tracking epidemic control strategies or issuing updates on future steps. Additionally, it also provides convenience for the general public and enables better decisions to be made in preventing infection.

\section{Conclusion}

The purpose of these online epidemic mapping services is to educate travellers and assist the public or to analyzing the spatial and current trends and patterns of COVID-19 for public health authorities in assessing/revising current control measures.

\section{Declarations}

\section{Acknowledgments}

The authors would like to thank WHO, Johns Hopkins University, and Baidu for their contribution to controlling the outbreak. This project was partly funded by the Natural Science Foundation of Hainan Province of China (Contract No. 318MS133) thanks to the Department of Science and Technology of Hainan Province.

\section{Authors' contributions}

manuscript review and contribute (Yijun Yang,Yingai Zhang); Study design and original writing (Yufang Cao); acquisition and apply of data and language processing and polishing (Rui Qiu); Information collection ( Chao Ji, Zhidian Wu).

\section{Ethics approval and consent to participate}

Not applicable.

\section{References}


1. Jianhui Wang, Hongbo Qi, Lei Bao, Fang Li, Yuan Shi. A contingency plan for the management of the 2019 novel coronavirus outbreak in neonatal intensive care units. Lancet Child Adolesc Health. 2020;4(4):258-259. https://doi.org/10.1016/

2. Jun Chen, Tangkai Qi, Li Liu, Yun Ling, Zhiping Qian, Tao Li, et al. Clinical Progression of Patients With COVID-19 in Shanghai, China. J Infect. 2020;80(5):e1-e6. https://doi:10.1016/ jinf.2020.0 3.004.

3. Chan JF, Yuan S, Kok KH, To KK, Chu H, Yang J, et al. A familial cluster of pneumonia associated with the 2019 novel coronavirus indicating person-to-person transmission: a study of a family cluster. 2020;15;395(10223):514-523. https://doi:10.1016/S0140-6736(20)30154-9.

4. Lidia Morawskaa, JunjiCaob. Airborne transmission of SARS-CoV-2: The world should face the reality. Environment International. 2020;10;139:105730. https://doi:10.1016/j.envint.2020.105730.

5. Shi Zhao, Qianyin Lin, Jinjun Ran, Salihu S Musa, Guangpu Yang,et al. Preliminary estimation of the basic reproduction number of novel coronavirus (2019-nCoV) in China, from 2019 to 2020: A datadriven analysis in the early phase of the outbreak.Int J Infect Dis. 2020;92:214-217. https://doi:10.1016/j.ijid.2020.01.050.

6. Chih-Cheng Lai, Tzu-Ping Shih, Wen-Chien Ko, Hung-Jen Tang, Po-Ren Hsueh. Severe acute respiratory syndrome coronavirus 2 (SARS-CoV-2) and coronavirus disease-2019 (COVID-19): The epidemic and the challenges.Int J Antimicrob Agents. 2020;55(3):105924. https://doi:10.1016/ ijantimicag.2020.105924.

7. Huihui Wang, Xuemei Li, Tao Li, Shubing Zhang, Lianzi Wang, et al. The genetic sequence, origin, and diagnosis of SARS-CoV-2. Eur J Clin Microbiol Infect Dis . 2020;39(9):1629-1635.https://doi: 10.1007/s10096-020-03899-4.

8. BoulosMN, Roudsari AV, Carson ER. Health Geomatics: An Enabling Suite of Technologies in Health and Healthcare (Methodolical Review). J Biomed Inform. 2001;34:195-219. https://doi:10.1006/jb 2001.1015.

9. Gańczak John Snow and cholera--the bicentenary of birth. Przegl Epidemiol. 2014;68:89-92, 16971.https://pubmed.ncbi. nlm.nih.gov/25004638.

10. Shaw N, McGuire S. Understanding the Use of Geographical Information Systems (GIS) in Health Informatics Research: A Review. J Innov Health Inform. 2017;24:940.https://doi:10.14236/jhi.v 940.

11. Boulos MN, Roudsari AV, Carson ER. Health Geomatics: An Enabling Suite of Technologies in Health and Healthcare (Methodolical Review). J Biomed Inform. 2001;34:195-219.https://doi: 10.1006/jbin.2001.1015.

12. Norström M, Geographical Information System (GIS) as a Tool in Surveillance and Monitoring of Animal Diseases. Acta Vet Scand Suppl. 2001;94:79-85.https://doi:10.1186/1751-0147-42-s1-s79.

\section{Figures}




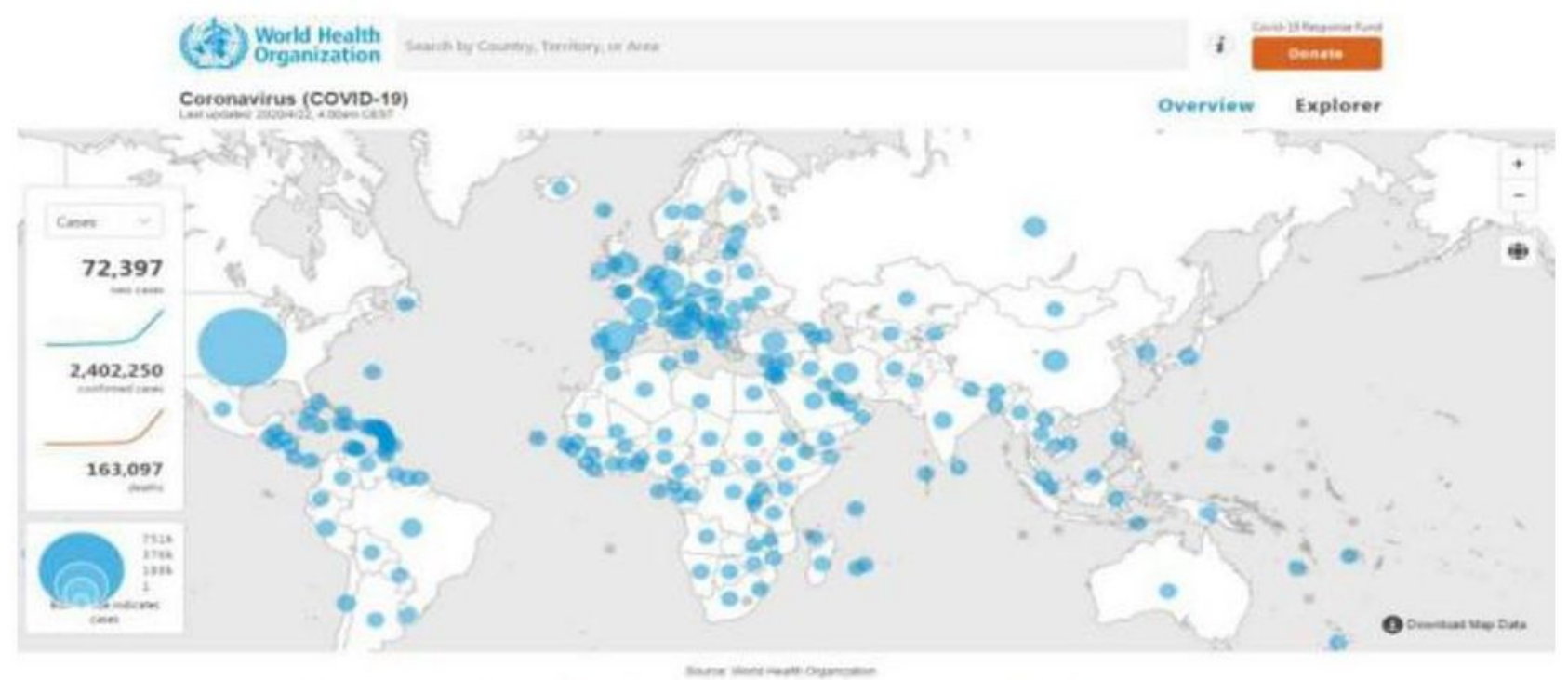

Globally, as of 2:00am CEST, 21 April 2020, there have been 2,402,250 confirmed cases of COVID-19, including 163,097 deaths, reported to WHO.

\section{Figure 1}

WHO world map of COVID-19. Web browser screenshot of the WHO world map of COVID-19 displaying data for each country affected by COVID-19 as of 16 April 2020. https://covid19. who.int/. As the cursor is moved over a country, a tool tip appears with the cumulative number of reported cases. Note: The designations employed and the presentation of the material on this map do not imply the expression of any opinion whatsoever on the part of Research Square concerning the legal status of any country, territory, city or area or of its authorities, or concerning the delimitation of its frontiers or boundaries. This map has been provided by the authors. 


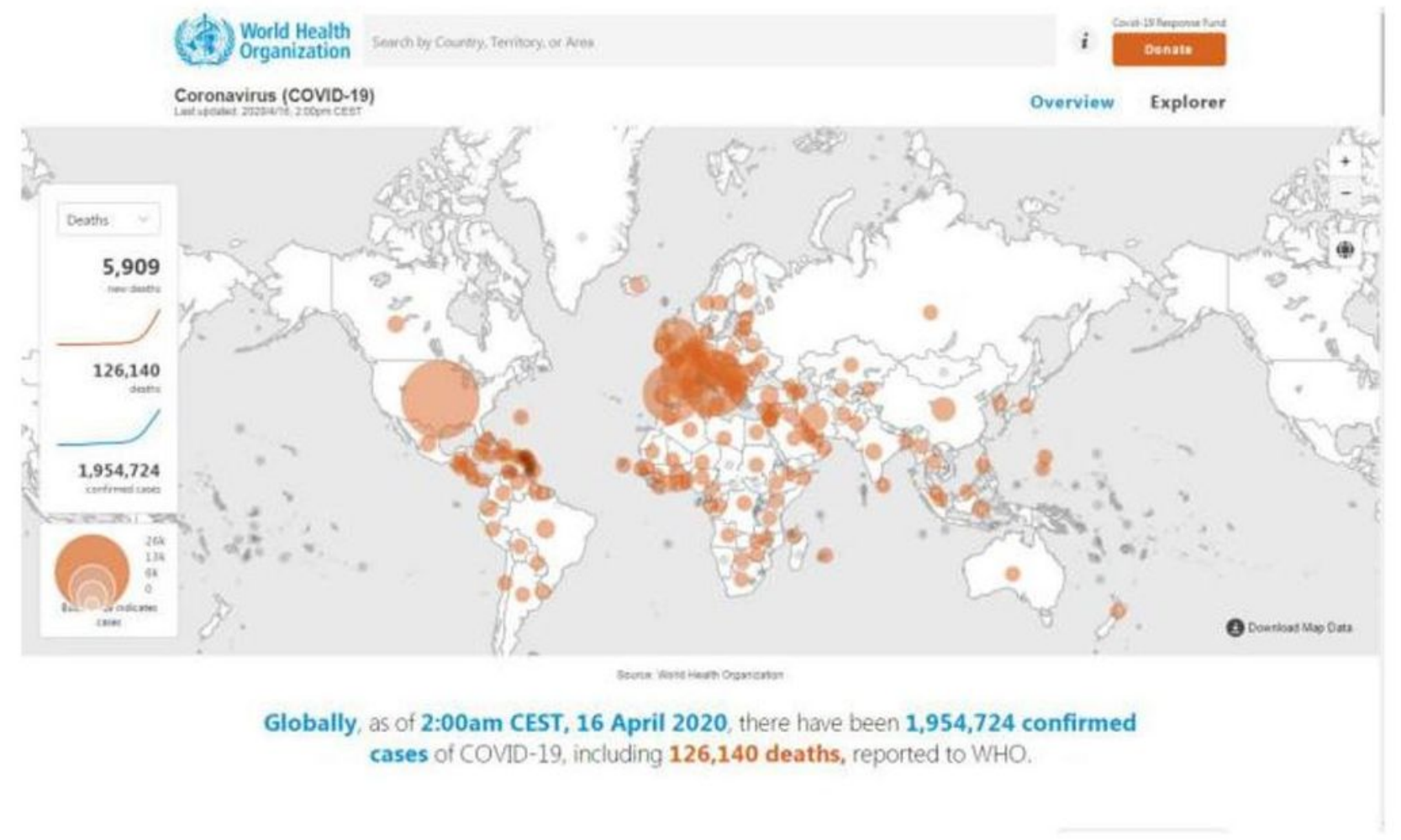

Figure 2

WHO world map of COVID-19. A web browser screenshot of the WHO's COVID-19 outbreak map shows the cumulative number of COVID-19 deaths in each country as of 16 April 2020. https://covid19.who.int/. As the cursor is moved over a country, a tool tip appears with the cumulative number of deaths. Note: The designations employed and the presentation of the material on this map do not imply the expression of any opinion whatsoever on the part of Research Square concerning the legal status of any country, territory, city or area or of its authorities, or concerning the delimitation of its frontiers or boundaries. This map has been provided by the authors. 


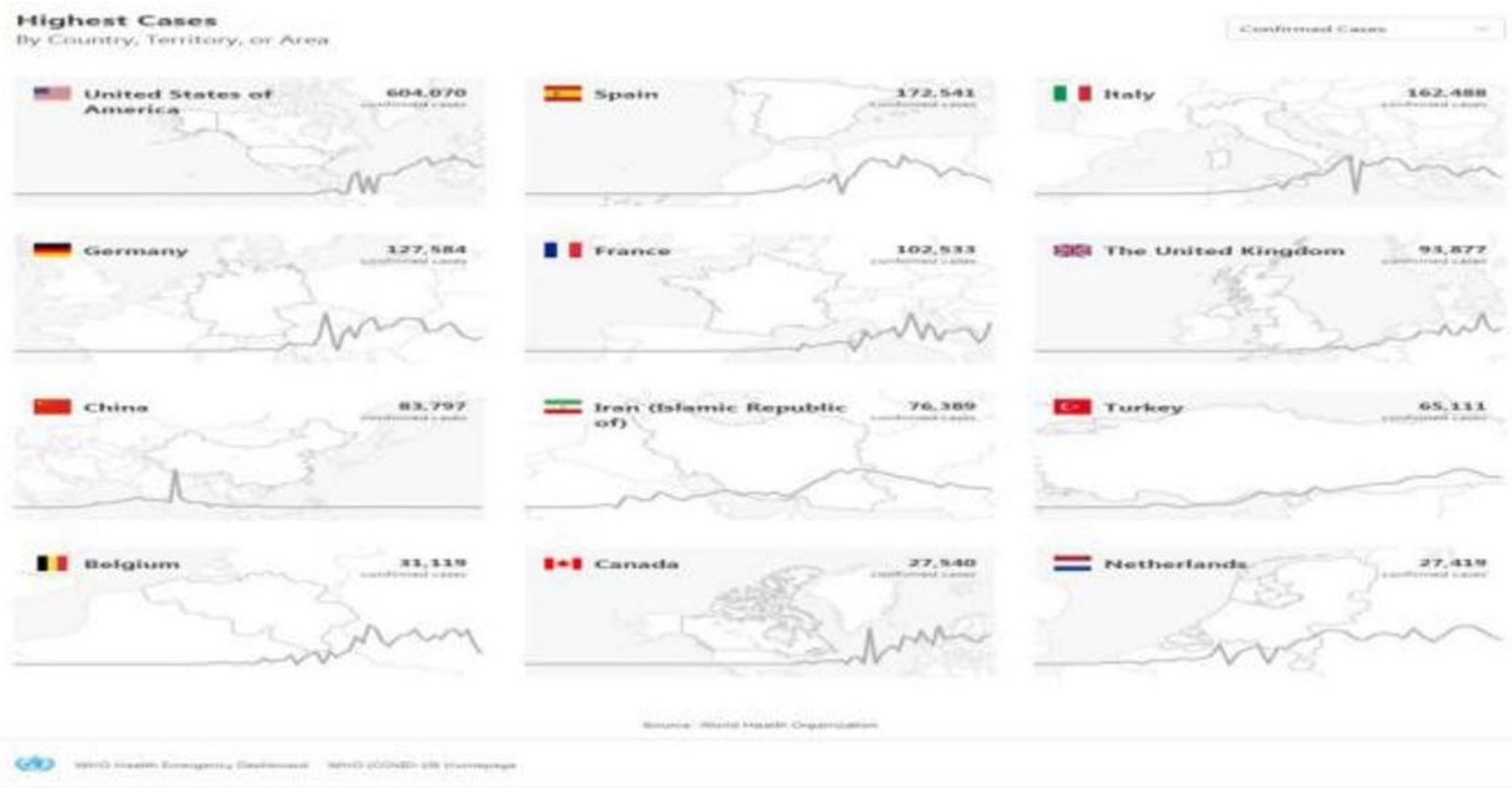

\section{Figure 3}

Based on the trend graphs divided by country or region, the number of confirmed cases on a certain day can be displayed by cursor selection and further clicking to enter the country to view the detailed epidemic situation. https://covid19.who.int/. Note: The designations employed and the presentation of the material on this map do not imply the expression of any opinion whatsoever on the part of Research Square concerning the legal status of any country, territory, city or area or of its authorities, or concerning the delimitation of its frontiers or boundaries. This map has been provided by the authors. 


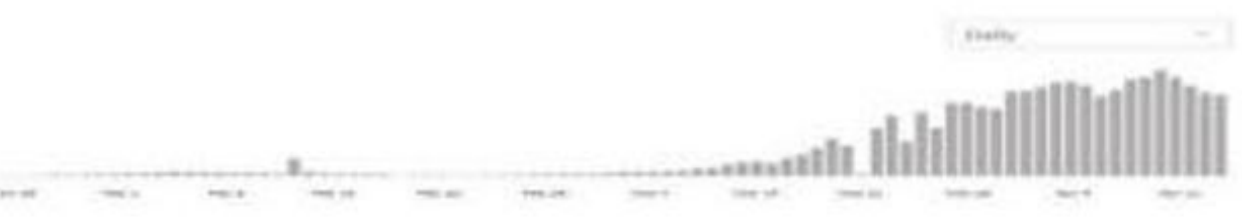

Denews Over rirve

\subsection{0}

seatin:

Case Compartsern

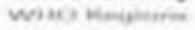

enesope

Anenteses

weserm ferithe

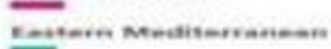

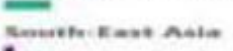

*

Afries

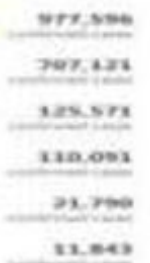

suans
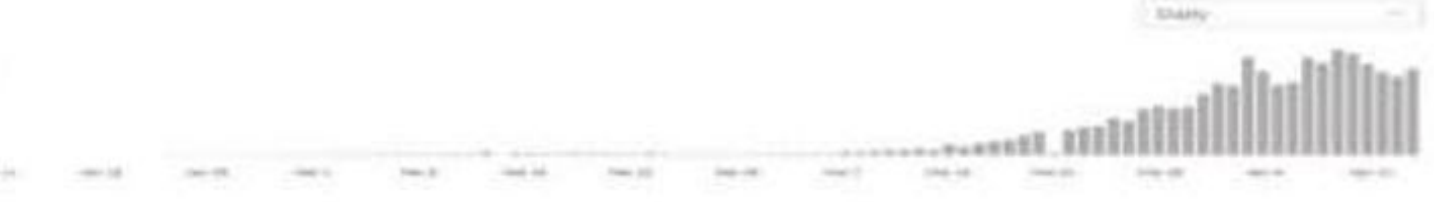

\section{Daily Casen}

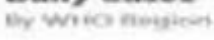

secrope

sxoes
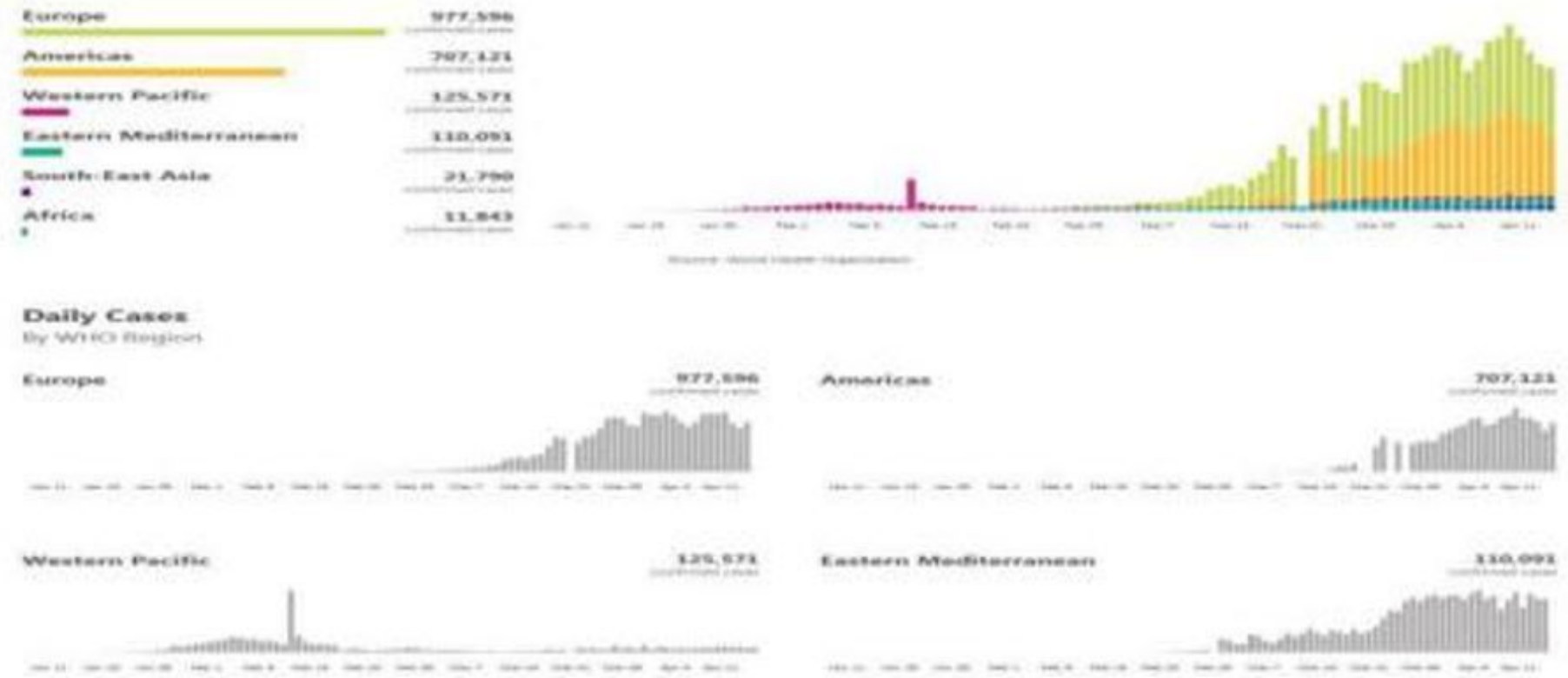

Amertens

noxians
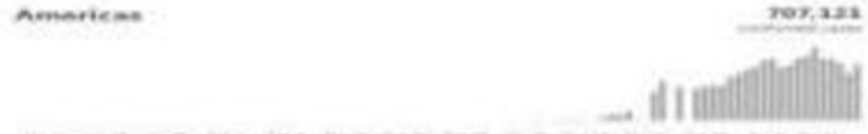

Seberto-Ean nuta
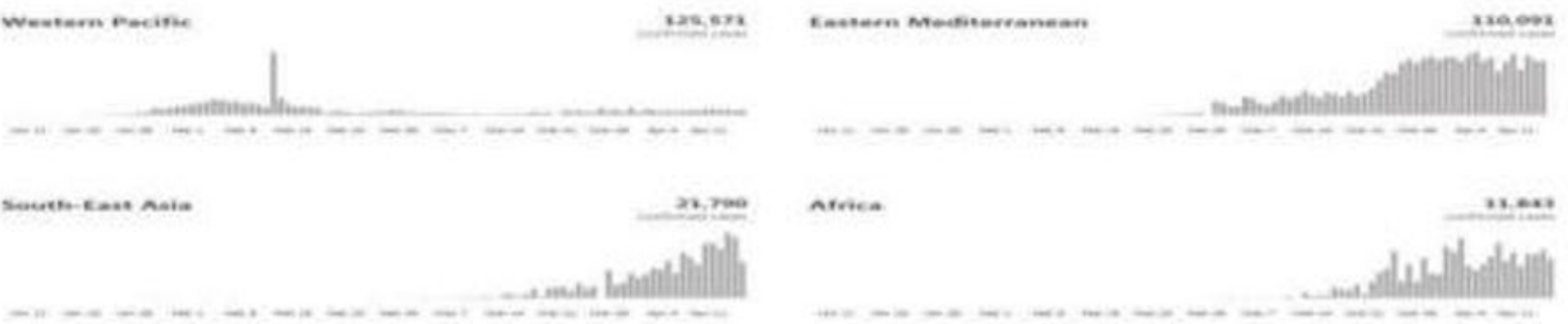

Arres.

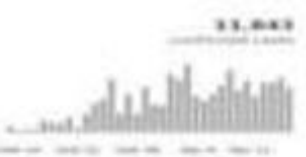

\section{Figure 4}

WHO has drawn a bar chart of the epidemic situation divided by country or region, and it can be selected by the cursor to display the number of confirmed cases and the number of deaths as well as the cumulative number of confirmed cases and deaths. 


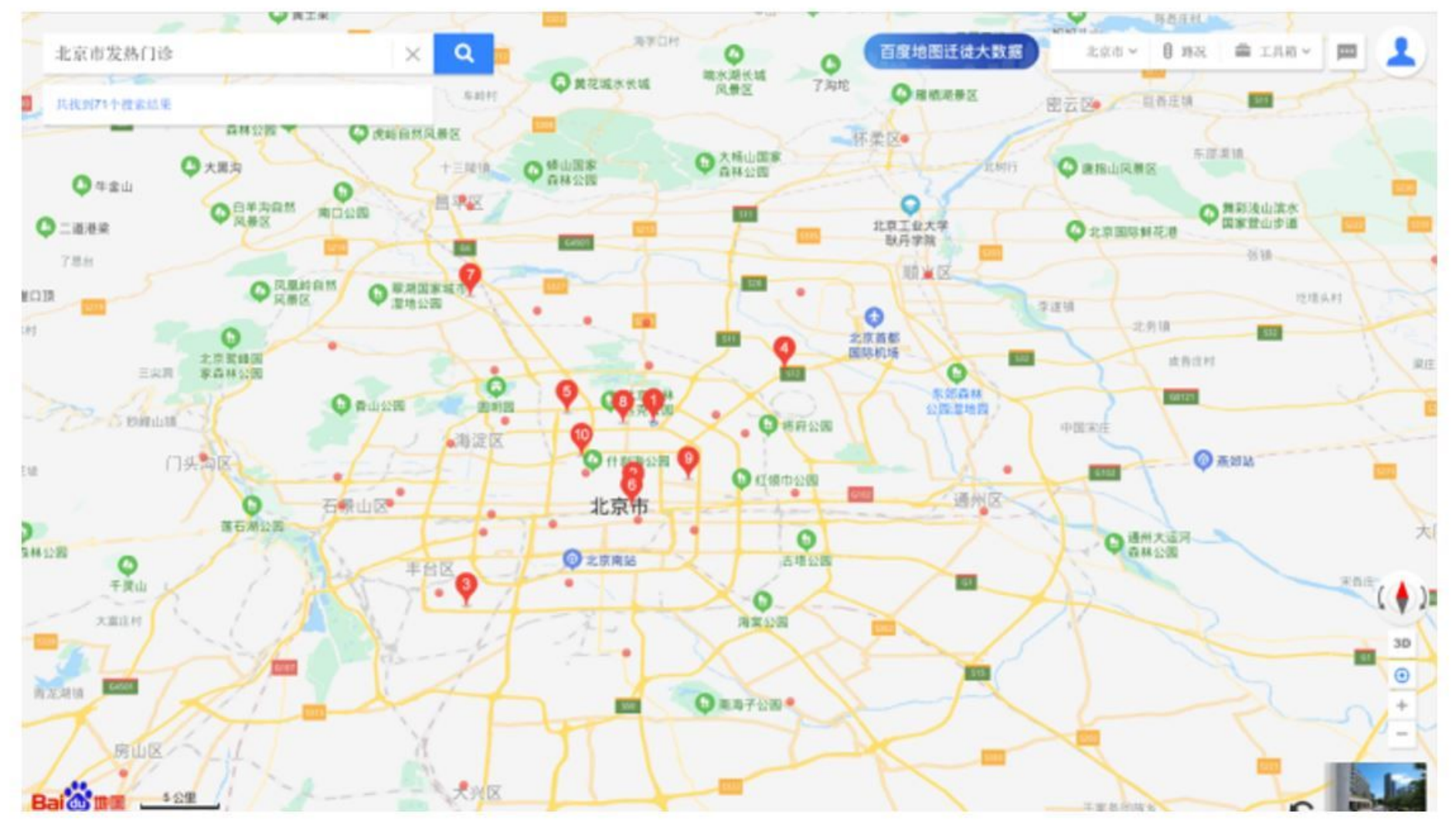

Figure 5

The graph shows the fever clinics in Beijing drawn by Baidu. The red data points indicate the locations of the fever clinics. People can choose the nearest fever clinic to ensure that they are diagnosed and treated as soon as possible. http://i7q.cn/6pXt24. 


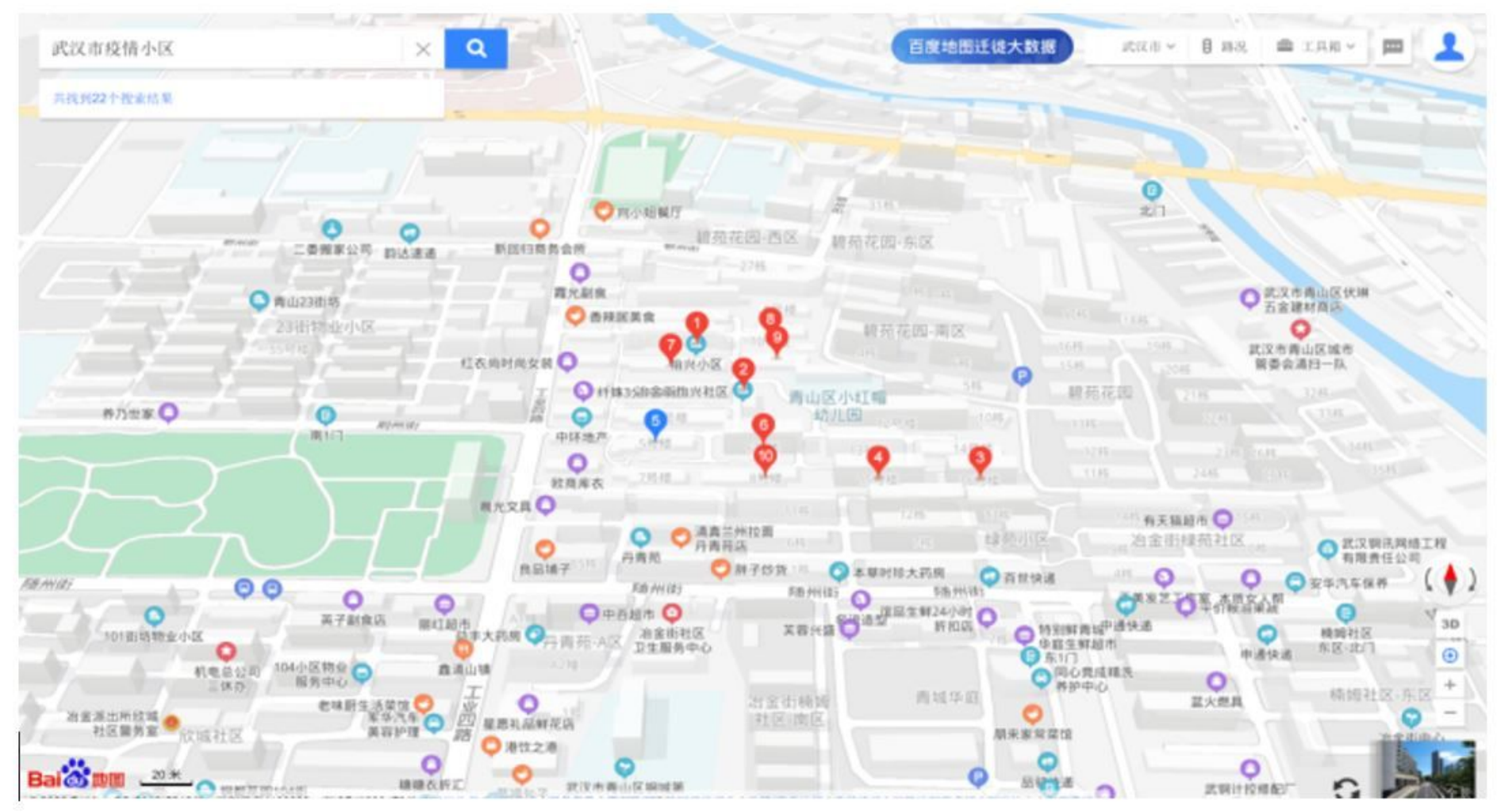

\section{Figure 6}

The picture shows a map drawn by Baidu of the "epidemic area" in Wuhan marked in red to warn the public of isolation and facilitate government response. http://i7q.cn/6xtFZv.

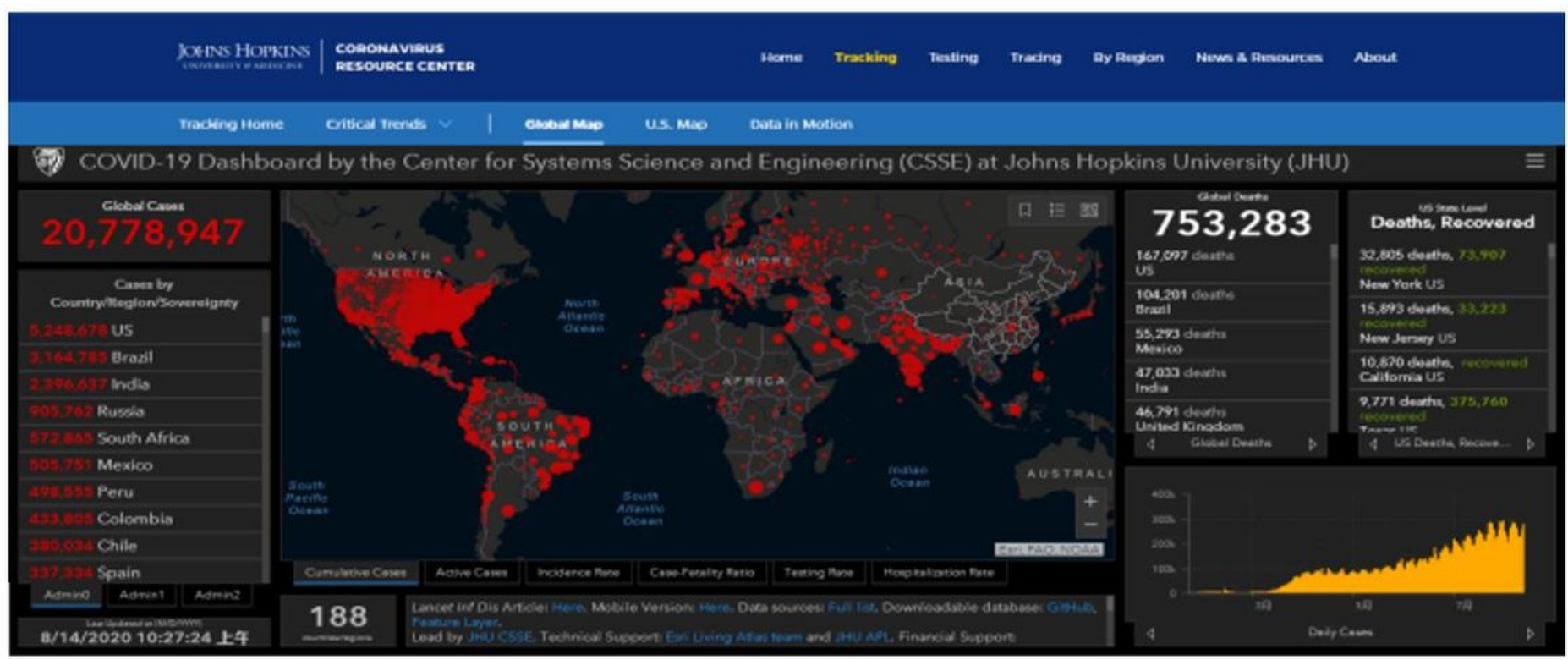

\section{Figure 7}

The picture shows a map drawn by JHU of the global "epidemic area" marked in red to warn the public of isolation and facilitate government response. https://coronavirus.jhu.edu/map.html. Note: The 
designations employed and the presentation of the material on this map do not imply the expression of any opinion whatsoever on the part of Research Square concerning the legal status of any country, territory, city or area or of its authorities, or concerning the delimitation of its frontiers or boundaries. This map has been provided by the authors. 